\title{
Status and prospects: personalized treatment and biomarker for airway remodeling in asthma
}

\author{
Jintao Zhang, Liang Dong \\ Department of Respiratory and Critical Care Medicine, Qilu Hospital, Cheeloo College of Medicine, Shandong University, Jinan, China \\ Contributions: (I) Conception and design: All authors; (II) Administrative support: None; (III) Provision of study materials or patients: None; (IV) \\ Collection and assembly of data: All authors; (V) Data analysis and interpretation: All authors; (VI) Manuscript writing: All authors; (VII) Final \\ approval of manuscript: All authors. \\ Correspondence to: Liang Dong. Department of Respiratory and Critical Care Medicine, Qilu Hospital, Cheeloo College of Medicine, Shandong \\ University, 107 Wenhuaxi Road, Jinan, China. Email: d15506@126.com.
}

\begin{abstract}
Airway remodeling, as a major characteristic of bronchial asthma, is critical to the progression of this disease, whereas it is of less importance in clinical management. Complying with the current stepwise treatment standard for asthma, the choice of intervention on the clinical status is primarily determined by the patient's treatment response to airway inflammation. However, a considerable number of asthmatic patients, especially severe asthmatic subjects, remain uncontrolled though they have undergone fortified anti-inflammation treatment. In the past few years, a growing number of biologics specific to asthma phenotypes have emerged, bringing new hope for patients with refractory asthma and severe asthma. While at the same time, the effect of airway remodeling on asthma treatment has become progressively prominent. In the era of personalized treatment, it has become one of the development directions for asthma treatment to find reliable airway remodeling biomarkers to assist in asthma phenotypes classification, and to further combine multiple phenotypes to accurately treat patients. In the present study, the research status of airway remodeling in asthma is reviewed to show the basis for classifying and treating such disease. Besides, several selected airway remodeling biomarkers and possibility to use them in individual treatment are discussed as well. This study considers that continuously optimized mechanisms and emerging biomarkers for airway remodeling in the future may further support individual therapy for asthma patients.
\end{abstract}

Keywords: Asthma; airway remodeling; biomarker; personalized treatment

Submitted Feb 25, 2020. Accepted for publication Aug 13, 2020.

doi: $10.21037 /$ jtd-20-1024

View this article at: http://dx.doi.org/10.21037/jtd-20-1024

\section{Introduction}

Asthma, as a highly prevalent respiratory disorder worldwide, refers to a chronic noncommunicable airway disease primarily manifested by airway inflammation, airway remodeling, as well as airway hyperresponsiveness. Recently, asthma has become progressively prevalent and currently affected about 334 million people worldwide (1). As suggested from the latest GINA (2), bronchial asthma is defined as a heterogeneous syndrome instead of a single disease. It exhibits varying symptoms and intensity over time with variable expiratory flow limitations. Note that for asthmatics exhibiting similar clinical manifestations, they may develop highly different endotypes and phenotypes, thereby adversely affecting their responses to treatment (3). Given the complexity of airway remodeling, existing studies on asthma phenotypes placed a major emphasis on airway inflammation (4).

Acting as a main feature of asthma, airway remodeling is critical to determine progression of the disease. Early detection aided by effective intervention to airway remodeling may down-regulate the incidence of acute asthma attacks and mitigate severe damage $(5,6)$. As fueled by the recent advancement of different assistive 
technologies, the outline of airway remodeling is emerging step by step. Under the background of personalized therapy, seeking effective biomarkers for airway remodeling classification and precise treatment of asthmatic subjects has become one of the future development directions for asthma treatment.

In this paper, we review the published literature on some of the potential biomarkers and therapy aiming at airway remodeling in asthma. The current status and encountered problems of airway remodeling research are also highlighted, in order to explore the possibility for personalized treatment of asthma based on airway remodeling.

\section{Research status of airway remodeling in asthma}

\section{Characteristics of airway remodeling in asthma}

Asthmatic airway remodeling was initially proposed in 1922 (7). Numerous studies on airway remodeling have been conducted or are ongoing. Multiple asthma studies based on human subjects have been conducted in depth, underpinning further expansion and clinical applications. Airway remodeling covers multiple cell interactions (8), which is primarily characterized by airway epithelial variations, reticular basement membrane (RBM) thickening, mucous glands hypertrophy, smooth muscle hyperplasia/ hypertrophy as well as angiogenesis. All of these variations in airway components interact with each other, eventually leading to adverse outcomes (e.g., airway wall thickening, epithelial dysfunction, and abnormal mucus secretion) (9). Compared with healthy subjects, the thickness of airways in asthmatic patients could increase significantly. Studies suggested that airway wall thickness in patients with fatal asthma increased by $50-300 \%$, and that of non-fatal asthma patients increased by $10-100 \%$ (10). Furthermore, the obstruction will be exacerbated by excessive mucus secretion and inflammatory hyperemia (11).

Studies reported airway remodeling existing in the early stages of asthma patients even before clinical manifestations (12). It was not just related to the progressive response to long-term persistent inflammation that was initially considered. It is noteworthy that even without other inflammations, mechanical stresses caused by bronchoconstriction may also induce airway remodeling $(13,14)$.

\section{Significance of airway remodeling research in astbma}

Previous studies reported that respiratory and airway function is not completely parallel to airway inflammation and may be related to airway remodeling (15). Similarly, other researchers considered that though there are overlapping links between airway inflammation and remodeling, the two should still pertain to different systems (16). Each of them has its own set of pathogenesis and affects each other over asthma development. Studies combining the analysis of inflammation and airway remodeling can provide more insights into the complete pathophysiology of asthma (17).

Besides overlapping with inflammatory mechanisms, airway remodeling is also related to airway hyperreactivity, whose persistence and exacerbation are partially attributed to some airway remodeling events (e.g., collagen deposition and increased airway smooth muscle mass and contractility) (18).

Remodeling can change airway structure and may gradually lead to fixed airway obstruction. For asthmatics, fixed airflow limitation may not act as a curable trait, whereas it is a feature that should be treated correctly to prevent overtreatment (19). Moreover, it has also been considered one of the vital features of numerous severe asthmatics (20). For health-care expenditure, though severe asthma only accounts for a small proportion of the asthma population, it assumes the majority of the medical expenses and social pressure while airway remodeling acts as a prominent trait for severe asthmatics $(21,22)$.

For children with asthma, multiple birth cohort studies reported that airway structural variations can take place in early childhood in parallel with permanent lung function damage, and that the reticulum basement membrane is considered a predictor of future asthma development, independent of airway smooth muscle thickness or eosinophilic inflammation (23). On the whole, airway remodeling is an imperative problem that should be faced and resolved.

\section{Research dilemmas in airway remodeling}

The complexity of airway remodeling is reflected by its involvements in different tissues and cell types, as well as its interrelationship with a range of biological processes. Its slow progress is mostly caused by many unknown factors of pathophysiological mechanisms and difficulties in clinical application (24).

As the most commonly used existing disease model, mice models are extensively used in asthma research (25), whereas it has many restrictions. First, mice cannot develop asthma naturally, and only some specific types of asthma can be 
induced artificially in mice. Second, mice are significantly different from humans in airway structure and size (26,27). Both of them have highly impacted the asthma research, especially for airway remodeling.

Biopsy is generally known as the most effective method to assess the extent of airway remodeling in asthma patients. Moreover, imaging examinations, especially CT, are also considered a useful way to assess airway remodeling in human asthma subjects. However, all of these techniques are limited by practicality and ethicality (28).

Moreover, the airway remodeling we have often observed and measured is confined to large and mediumsized airways for the finite of technical level. However undoubtedly, bronchioles and other smaller bronchus can also participate in this progression. Airway remodeling may have different effects on the smaller ones (29) for their distinct components and structures. However, it is difficult to investigate airway remodeling.

Besides, existing studies on asthma were primarily conducted using cross-sectional methods (30), whereas the prominent feature of airway remodeling is that it is a longitudinal process involved long-term evolution, which also adds obstacles to airway remodeling research.

\section{Basic research for airway remodeling}

Though airway remodeling has several adverse effects as mentioned on human subjects, causing decreased lung function and persistent and progressive airflow limitation, it indeed has some benefits. For asthma patients, airway remodeling could inhibit airway contraction and counteract excessive narrowing $(18,31)$. Thus far, the complete mechanism of airway remodeling remains unclear, and it is unwise to blindly intervene airway remodeling. How to prevent the pathological airway remodeling effectively and return it to the track of reasonable repair smoothly now is the lead object of existing asthma studies. Similar to many existing effective approaches to manage diseases, successful management of asthma is also determined by the insights into its basic biological processes and dysfunction in the context of disease (32).

Over the past few decades, airway ecological characteristics of asthmatics have been further explored (33), and different mechanisms and cellular interactions in asthma have been extensively delved into (e.g., autophagy and senescence) (34,35). As the basic research has been deepening, the whole framework of remodeling mechanisms in asthma continues to be perfect.
Epidemiological studies reported that both genetic and environmental factors can reduce lung function and increase the likelihood of airway remodeling (36). In the field of asthma-associated gene, genetic researches related to airway remodeling have been rarely conducted. Though over 100 genes have already been proved related to asthma, only few of them are linked to decreased lung function of asthma (37). It has been demonstrated that three genes variants, namely, IL-13, PLAUR and CHI3L1 (38-40), are associated with airway remodeling in asthma.

Among various asthma animal models, equine asthma models have also aroused more attention from zoologists in recent years. Compared with mice, horses can spontaneously produce asthma, while its larger respiratory system is also easy to observe and research for airway remodeling. Besides, isolating inflammation and airway remodeling may be even likely to take place in asthma equine models (17), making equine model an appropriate model to study non-eosinophilic asthma.

Considerable puzzles remain in airway remodeling, and more intensive basic studies are still required to demonstrate whether these experiments are performed longitudinally or horizontally. These are necessary to provide more insights into the entire process and subsequently the choice for treatment methods.

\section{Advanced assistive technologies for airway remodeling}

In the past, for the complexity of airway remodeling involving the chain reaction of multiple cellular molecules at different periods and the limitations of multiple detection technologies, it has always been difficult to explore it. Even biopsy is a reliable method for diagnosis of airway remodeling, as an invasive detection technique, its function is limited. Now optimized assistive technologies, including advances in imaging technology and measurement indicators (41-43), can be adopted as a feasible way to delve into and exploit this character.

Spirometry is currently the gold standard for the diagnosis of asthma, but it is short in intuitively reflecting structural airway changes (44). CT is an important technology to diagnose multiple respiratory diseases; it is also conducive to measuring the degree of airway remodeling. In previous studies, fractional exhaled nitric oxide (FeNO), as one of the non-injury markers reflecting chronic inflammation in asthma patients, was considered unrelated to the airway thickness in asthma patients (45). After accurate sub-generation of bronchial trees, a three- 
dimensional CT analysis was conducted by researchers (46), and the results indicated that $\mathrm{FeNO}$ in asthma patients was associated with thickening of bronchial walls in the third to the sixth generation. It is therefore suggested that FeNO might be useful in assessing airway structure variations in asthma patients, especially in the distal airway. Moreover, some researchers conducted high-resolution CT studies and demonstrated that airway remodeling in asthma was more significant in the distal airway and subbronchial lobes; thus, it was reported that airway remodeling could help predict small airway involvement and identify targets for local treatment of asthma, as well as serve as a predictor of early asthma $(47,48)$. Another report (49) also proposed a novel concept for assessing airway in CT. It was revealed that in quantitative CT (qCT), calculating the percentage of tracheal cavity area, Delta Lumen, could indicate adverse outcomes and airway remodeling in asthma patients. With the development of CT, more insights into airway remodeling have been gained.

Apart from CT, there are many other assistive technologies assisting in evaluating airway remodeling. Adams et al. (50) used a birefringent fiber platform to observe airway smooth muscle in vivo. Meantime, they improved the imaging ability to observe the structure and function of airway smooth muscle in asthma patients. Replacing the conventional tissue staining method by high-resolution nonlinear optical microscopy (NLOM), researchers tested the lungs of asthmatic patients and control and reported that the levels of disordered and broken fibrous collagen in the airway remodeling extracellular matrix (ECM) of asthmatic patients were elevated, further enriching the relevant characteristics of airway remodeling (42).

Since airway remodeling can change the respiratory tract not only in quantity but in property (51), airway remodeling is still being roughly measured even under the support of multiple technologies. However, it cannot be disclaimed that following the introduction of novel parameter and progress of technologies, obstacles ahead are being continuously reduced.

\section{Airway remodeling biomarkers in asthma}

The proper classification of asthma by biomarkers or any other means is the premise of individualized treatment. In recent years, biomarkers have aroused increasing attention in the diagnosis and control of diseases, including diabetes and cancer (52). It is currently known that asthma-related biomarkers can be obtained in various sources (e.g., urine, blood, bronchoalveolar lavage fluid, induced sputum, exhaled gas agglutination, and bronchial biopsy). For minimal invasion and reproducibility, blood and exhaled breath may show more advantages over others $(41,53)$. In asthma, especially severe asthma, several biomarkers already available for clinical practice including blood eosinophil, sputum eosinophil and neutrophil, serum total $\mathrm{IgE}$ and $\mathrm{FeNO}$, which could reflect underlying airway inflammation (53).

\section{Significance of airway remodeling markers}

Though considerable efforts have been paid to verify the importance of airway remodeling in asthma, the lack of accessible and valid biomarkers for clinical application has also become a major problem hindering the progress of airway remodeling research (17), the emphasis of most biomarkers is still placed on the inflammatory response in this disease. As one of the causes of chronic irreversible damage in asthma, airway remodeling exerts extensive and far-reaching impact on asthma patients.

For low T2 asthma, it was reported that patients with T2 low asthma usually lack a history of environmental allergies and less responsive to corticosteroids (54). For the absence of effective biomarkers, exclusion methods have been generally used to support diagnosis of low T2 asthma (55). In this case, biomarkers reflecting airway remodeling may be conducive to identifying this phenotype.

\section{Exploration of airway remodeling marker in asthma}

The current overlaps of biomarkers reveal that distinguishing asthma phenotype may require not one but a group of biomarkers (56) to accurately classify the endotype of asthma. Many proteins and cytokines have been confirmed to be associated with airway remodeling in asthma (e.g., periostein), which has been extensively studied in recent years (8). It is inevitable that their remodeling function mostly overlaps with inflammation. Besides, for many limitations, the level of airway remodeling in human-based studies is usually reflected in the response to treatment (e.g., improvement in lung function and FEV1) (57-59). Though researchers have confirmed the correlation between the variations in lung function and airway remodeling (60), these variations are indirect and ambiguous. It is difficult to effectively analyze and estimate the level of remodeling via these methods, while patients' remodeling phenotypes are more difficult to classify. 
Furthermore, some scholars considered that imaging technologies can also act as markers of airway remodeling, including the application of qCT and ultra-polarized nuclear magnetic resonance imaging (60). Researchers can even use imaging methods to classify asthma patients and guide further treatment (61). However, imaging technologies are so roughly to observe and calculate the airway structure and cannot accurately distinguish airway hierarchy. More importantly, their applications in asthma are also confined by practicality and economy.

\section{Potential airway remodeling biomarker}

Multiple Studies based on bronchoalveolar lavage and endobronchial biopsies have proposed plenty of feasible biomarkers (e.g., matrix metalloproteinase-9, proteaseactivated receptor 2 and even its ligands (62-64) that are related to airway remodeling). However, most of the mentioned molecules should be collected from biopsy or alveolar lavage fluid, which is invasive and labor-intensive (65), thereby limiting their clinical applications. Unlike others, there are three potential molecules, namely, galectin-3 (Gal-3), YKL40, and VitD. It has been proven by biopsy that these molecules in blood can partially reveal the change of airway structure, revealing that they may be practically used to support the diagnosis of airway remodeling.

\section{Gal-3}

Gal-3 that can be secreted and measured in the blood is a shared factor in fibrosis formation in different organs (66). Thus, it has been considered a biomarker, associated with many diseases (e.g., heart failure) (67). In animal model, the Gal-3 knockout mice have been successfully established. Following experiments validated that Gal-3 could promotes airway remodeling via secretion of multiple cytokines as well as recruitment of inflammatory cells (68). After analyzing the RBM thickness, eosinophil and neutrophil infiltration, and proteomics characteristics of eight severe asthmatic patients via bronchoscopy biopsies, researchers reported that Gal3 as a biomarker could effectively reflect the reduction of bronchial smooth muscle (BSM), eosinophilic inflammation and muscle protein component in asthmatics $(69,70)$. However, for the small sample size, the results have many limitations, which require further experimental verification. Additionally, as a pro-fibrotic molecule Gal-3 is also a biomarker of heart disease and fibrosis. The level of Gal-3 in serum may correlate with inflammation and other fibrosis processes as well, independent of remodeling in asthma (71).

\section{YKL-40}

As a member of the mammalian chitinase-like protein family, YKL-40 refers to an inflammatory glycoprotein whose exact function remains unclear (72). Now, it is considered involved in many pathophysiological processes (e.g., inflammation, injury and tissue remodeling) (73). Previous research confirmed that CHI3L1, the gene encoding YKL-40 protein, was associated with airway remodeling in asthma (38). Bara et al. (74) conducted studies on airway biopsy samples from 40 asthmatic patients and reported the close relationship between YKL-40 and airway remodeling. The results showed that YKL-40 can promote BSM cell proliferation and migration by PAR-2-dependent pathway. In addition, the expression of YKL-40 in epithelial was positively associated with BSM mass in asthma. In serum sample, Konradsen et al. (75) compared serum YKL-40 levels in children with therapy-resistant asthma $(n=34)$, controlled persistent asthma $(n=36)$ and healthy control $(\mathrm{n}=27)$. In children with therapy-resistant asthma serum YKL-40 levels were at the relatively high level and closely related to airway thickness and asthma control. Above all, through biochemical and immunohistochemical analysis, Chupp et al. (76) found that YKL-40 levels in serum were correlated with the thickness of airway subepithelial basement membrane. Similar to Gal-3, YKL40 also increased in other fibrotic diseases like idiopathic pulmonary fibrosis (77).

\section{VitD}

Within the last few years, vitamin $\mathrm{D}$ has aroused huge attention from the scientific communities (78). A number of respiratory diseases, including asthma have now are related to vitamin D deficiency. Retrospective studies have shown that some trials support the beneficial role of vitamin D supplements in reducing the severity of asthma in children (79). It was speculated that vitamin $\mathrm{D}$ may improve airway inflammation and remodeling of asthma through multiple immune pathways including inhibition of growth of BSM cells through growth factor-induced phosphorylation of retinoblastoma protein and checkpoint kinase 1 (80). Studies (81) suggested that children with treatment-resistant severe asthma have significantly lower serum $25(\mathrm{OH}) \mathrm{D} 3$, the main circulating form of vitamin $\mathrm{D}$, than children with mild to moderate asthma. In the subsequent study, researchers collected airway biopsies and confirmed that lower 25(OH)D3 levels in patients' serum were associated with increased airway smooth muscle mass, poor lung function as well as asthma control. Furthermore, 25(OH)D3 levels were even unrelated 
to any other parameters of airway remodeling (e.g., airway inflammation). Despite its advantages, 25(OH)D3 also has many disadvantages. Previous studies have found that serum 25(OH)D3 levels may be affected by gender, age, season and several chronic disease $(82,83)$.

Given considerable restrictions, most studies on airway remodeling biomarkers in asthma have been limited to small samples. For airway remodeling, the variations of airway structure, some results derived from sputum other than serum may be more convincing, whereas the relative studies have been rarely conducted. At all events, more studies are still required for further demonstration.

\section{Asthma treatment and airway remodeling}

Though the phenotype and endotype of asthma have aroused increasing attention, anti-inflammatory treatment remains the pillar of asthma treatment. The association between existing treatment methods and endotype or phenotype remains insignificant in asthma. Antiinflammatory therapy for asthma has been gradually standardized and improved on clinical practice, while airway remodeling is considered the least affected by current pharmacological and biological treatments (24). Such significant gap provides the more possibility for future development.

\section{Effects of present treatment methods on airway remodeling}

\section{Glucocorticoid}

Glucocorticoid was demonstrated as the major drugs in current asthma therapy that can obviously ameliorate inflammation, whereas its impact on preventing or reversing airway remodeling remains under discussion. One study enrolled 45 corticosteroid-naive patients with persistent asthma and 28 healthy controls and conducted helical computed tomography. Their results reported that glucocorticoid can partially enhance lung function and decrease the thickness of the airway in asthma patients (84). However, many other opinions suggest that glucocorticoid cannot or slightly impact airway remodeling $(85,86)$, probably because of their different research object, drug dose, administration period and the size of the sample. However, to make matters worse, several studies revealed that inhaled glucocorticoids might have deleterious effects on airway epithelium (e.g., inducing apoptosis and impairing epithelial migration), thereby inhibiting its therapeutic effect for considerable aspects of asthma (87).

\section{Monoclonal antibodies}

A wealth of clinical trials regarding monoclonal antibodies suggested that a variety of IL-5 monoclonal antibodies (e.g., mepolizumab and reslizumab) can facilitate airway remodeling in asthma, including reducing the expression of ECM protein in the RBM, thereby partially restricting airway remodeling (48). For patients with long-term asthma with persistent airway obstruction, however, the current biologics have limited effects $(28,88-90)$. Though largescale clinical trials have suggested that the new monoclonal antibodies against IL-4/IL-13, [e.g., dupilumab and tralokinumab $(91,92)$ ] can effectively reduce the onset of asthma and improve FEV1, their exact effects on airway remodeling require further research. Studies (93) showed that IL-33 can increase RBM thickness in children with severe therapy resistant asthma (STRA) which was proven by endobronchial biopsies. After steroid treatment, IL-33 alone appears to be sufficient to sustain remodeling in the absence of IL-13. In general, IL-33 may act as a direct target for asthma treatment in the future due to its impact on reducing airway remodeling in STRA.

\section{Bronchial thermoplasty}

Bronchial thermoplasty brings emerging hope to treat airway remodeling in asthmatics. As a novel nondrug intervention, bronchial thermoplasty can impact the airway through thermal energy. Such technique significantly improves the patient's symptoms and quality of life (94) primarily by reducing excess airway smooth muscle, which has achieved promising results in clinical practice (95). It has proved that BT has slight variations in airway epithelial, mucinous gland and goblet cell hypertrophy or hyperplasia (96). However interestingly, some studies reported that the reduction of airway smooth muscle alone is not sufficient to explain its clinical results; they also consider that the reduction of airway smooth muscle mass cannot be completely caused by direct acute heating (97). For this novel technology, its mechanism of action to reduce the incidence of adverse reactions and improve patient outcomes requires further investigation.

Furthermore, other drugs [e.g., LAMA and LABA $(98,99)]$ have also been shown to improve airway remodeling, whereas they have also only been performed in animal models of asthma. Besides, there have been no data assessing outcomes of airway remodeling in asthmatics treated with these drugs.

Though the exact effect of current asthma treatment 
methods on airway remodeling requires further studies, it is undeniable that for multiple chronic airway diseases including asthma (67), more advanced and appropriate treatments are imperative to satisfy the requirement for this permanent airway injury.

\section{Prospect of airway remodeling therapy}

\section{Calcium receptor antagonist}

Gallopamil, a calcium receptor antagonist, has been demonstrated in vitro experiments to be able to inhibit airway smooth muscle cell proliferation $(100,101)$. A 12 -month double-blind controlled clinical trial was conducted (100), and the results proved that gallopamil could reduce the thickness of airway smooth muscle in patients with severe asthma without affecting the inflammation. As a result, the acute attack of asthma could be reduced, and the treatment of airway remodeling can be more effectively achieved.

\section{Antihistamine}

Released from mast cells, histamine has been thought to play an important role in the pathogenesis of allergic asthma (102). Previous research has shown conflicting results as to whether antihistamines are good or bad for asthma (103). Kunzmann et al. (104) reported that histamine can induce the expression of connective tissue growth factor CTGF in cultured lung fibroblasts and up-regulate the occurrence of chronic airway remodeling. Meantime, studies experimentally proved that (105) antihistamines exerted significant relief effect on asthma control and could enhance lung function. Accordingly, a theoretical possibility is provided for the use of antihistamines in asthma to some extent.

\section{Leukotriene receptor antagonist}

Given orally, montelukast is the most clinically-used leukotriene receptor antagonist in treating asthma. It has been proven that Montelukast ameliorated symptoms, rescue medication use and pulmonary function, and reduced the rate of exacerbation in asthmatics (106). For airway remodeling, limited evidence demonstrated the effectiveness of leukotriene inhibitors for the treatment or prevention of airway remodeling (107). Montelukast was also found to be able to inhibit the function of airway fibroblasts in mild asthma patients (108), and it might act as an effective method to facilitate airway remodeling and mitigate airway inflammation.

\section{Prostaglandin D type 2 (PGD2) receptor antagonist}

Existed evidence verified that PGD2, which acts on the PGD2 receptor 2 (DP2) have an important role in mediating eosinophilic airway inflammation in asthma and might be a promising target for the treatment of asthma (109). In a randomized placebo-controlled trial, researchers reported, as DP2 antagonist, fevipiprant could induced airway smooth muscle mass in bronchial biopsies from asthmatics (110). They also confirmed suppressed airway smooth muscle migration via using DP2-specific antagonists in an airway smooth muscle cell culture model (110). However, fevipiprant failed to improve lung function effectively in phase 3 trial.

\section{Possibility of personalized therapy for airway remodeling}

For many years, asthma treatment was primarily based on a "one treatment fits all" principle and the disease situation is not under control especially in some severe subjects (55). In the era of individualized therapy, treatment strategy according to different phenotypes of asthma can maximize the control of diseases. Gaining the insights into the degree and classification of airway remodeling may contribute to prevention and treatment of asthma.

As mentioned above, after years of research, the current research on airway remodeling has made significant progress, but having some distance from the practical application. Individualized treatment considers the patient as a whole and takes many factors including the patient's psychology, economy and other aspects into account not just the disease and the corresponding pathological mechanism (111). However, the complete mechanism of asthma especially airway remodeling has not been elucidated, which is the first step. Our understanding of the mechanism of airway remodeling in asthma is still in its infancy and remains to develop.

Different patients have unique remodeling phenotypes, which to some extent makes them respond to drugs differently, and their distinct phenotypes should be identified and precisely assessed for individualized treatment (8). Finding several reliable characteristics or biomarkers that can accurately distinguish phenotypes of patients lays the foundation for applying individualized treatment practically. Recently, researchers (112) developed a novel classification method for asthma which based on topological data analysis. They perform multidimensional data analysis to incorporate multiple features of airway remodeling, and successfully 
split asthma patients into four pathotypes. This analysis method further enriches the contents of each phenotype of asthma. At present, in the absence of effective airway remodeling biomarkers, such unusual method classifies the phenotype of asthma across the board and makes it possible for the next step of individual treatment.

Therapeutically, the improvement is made from having only one monoclonal antibody to a set of biologics with various targets and functions, and we will have to select some of them to make the right clinical decision (113). This further emphasizes the significance of searching for effective biomarkers and further reasonably performing asthma phenotyping to ascertain the correct treatment for the respective patient.

\section{Conclusions}

Thus far, there are still lots of puzzles in research about airway remodeling. Identification of ideal airway remodeling biomarkers in asthma remains inconclusive not to mention utilization. With the emerging of increasing biologics, compared with finding some novel and reliable biomarkers, further exploring the complete mechanism of airway remodeling is still in the first place. During the period of the progressively improvement on pathophysiologic mechanisms, suitable airway remodeling markers will gradually appear. When achieving asthma control, precise classification based on the biomarks or other methods should be achieved. On that basis, an ideal equilibrium state can be finally achieved.

\section{Acknowledgments}

Funding: This work was supported by grants from the National Natural Science Foundation of China (81770029), National Key Research and Development Project (2017YFC1310601) and Key Research Project of Shandong Province (2017GSF218056, 2020SFXGFY03-1, 2020SFXGFY03-3, 2020SFXGFY04).

\section{Footnote}

Conflicts of Interest: Both authors have completed the ICMJE uniform disclosure form (available at http://dx.doi. org/10.21037/jtd-20-1024). The authors have no conflicts of interest to declare.

Ethical Statement: The authors are accountable for all aspects of the work in ensuring that questions related to the accuracy or integrity of any part of the work are appropriately investigated and resolved.

Open Access Statement: This is an Open Access article distributed in accordance with the Creative Commons Attribution-NonCommercial-NoDerivs 4.0 International License (CC BY-NC-ND 4.0), which permits the noncommercial replication and distribution of the article with the strict proviso that no changes or edits are made and the original work is properly cited (including links to both the formal publication through the relevant DOI and the license). See: https://creativecommons.org/licenses/by-nc-nd/4.0/.

\section{References}

1. Papi A, Brightling C, Pedersen SE, et al. Asthma. Lancet 2018;391:783-800.

2. Global Initiative for Asthma. Global strategy for asthma management and prevention. 2020. Available online: www. ginasthma.org

3. Kaur R, Chupp G. Phenotypes and endotypes of adult asthma: moving toward precision medicine. J Allergy Clin Immunol 2019;144:1-12.

4. Simpson JL, Scott R, Boyle MJ, et al. Inflammatory subtypes in asthma: assessment and identification using induced sputum. Respirology 2006;11:54-61.

5. Martinez FD. Present and future treatment of asthma in infants and young children. J Allergy Clin Immunol 1999;104:169-74.

6. Saglani S, Payne DN, Zhu J, et al. Early detection of airway wall remodeling and eosinophilic inflammation in preschool wheezers. Am J Respir Crit Care Med 2007;176:858-64.

7. Huber HL, Koessler KK. The pathology of bronchial asthma. Arch Intern Med 1922;30:689-760.

8. Guida G, Riccio AM. Immune induction of airway remodeling. Semin Immunol 2019;46:101346.

9. Holgate ST. Epithelium dysfunction in asthma. J Allergy Clin Immunol 2007;120:1233-44; quiz 1245-6.

10. Elias JA, Zhu Z, Chupp G, et al. Airway remodeling in asthma. J Clin Invest 1999;104:1001-6.

11. Jeffery PK. Remodeling in asthma and chronic obstructive lung disease. Am J Respir Crit Care Med 2001;164:S28-38.

12. Royce SG, Cheng V, Samuel CS, et al. The regulation of fibrosis in airway remodeling in asthma. Mol Cell Endocrinol 2012;351:167-75.

13. Kariyawasam HH, Aizen M, Barkans J, et al. Remodeling 
and airway hyperresponsiveness but not cellular inflammation persist after allergen challenge in asthma. Am J Respir Crit Care Med 2007;175:896-904.

14. Grainge CL, Lau LC, Ward JA, et al. Effect of bronchoconstriction on airway remodeling in asthma. $\mathrm{N}$ Engl J Med 2011;364:2006-15.

15. Perry R, Braileanu G, Palmer T, et al. The economic burden of pediatric asthma in the United States: literature review of current evidence. Pharmacoeconomics 2019;37:155-67.

16. Fehrenbach H, Wagner C, Wegmann M. Airway remodeling in asthma: what really matters. Cell Tissue Res 2017;367:551-69.

17. Bullone M, Lavoie JP. The equine asthma model of airway remodeling: from a veterinary to a human perspective. Cell Tissue Res 2020;380:223-36.

18. Boulet LP. Airway remodeling in asthma: update on mechanisms and therapeutic approaches. Curr Opin Pulm Med 2018;24:56-62.

19. Pavord ID, Beasley R, Agusti A, et al. After asthma: redefining airways diseases. Lancet 2018;391:350-400.

20. Uwaezuoke SN, Ayuk AC, Eze JN. Severe bronchial asthma in children: a review of novel biomarkers used as predictors of the disease. J Asthma Allergy 2018;11:11-8.

21. O'Neill S, Sweeney J, Patterson CC, et al. The cost of treating severe refractory asthma in the UK: an economic analysis from the British Thoracic Society Difficult Asthma Registry. Thorax 2015;70:376-8.

22. Jarjour NN, Erzurum SC, Bleecker ER, et al. Severe asthma: lessons learned from the National Heart, Lung, and Blood Institute Severe Asthma Research Program. Am J Respir Crit Care Med 2012;185:356-62.

23. Saglani S, Bush A. Onset of structural airway changes in preschool wheezers. a window and target for secondary asthma prevention? Am J Respir Crit Care Med 2015;192:121-2.

24. Prakash YS, Halayko AJ, Gosens R, et al. An Official American Thoracic Society Research Statement: current challenges facing research and therapeutic advances in airway remodeling. Am J Respir Crit Care Med 2017;195:e4-19.

25. Kianmeher M, Ghorani V, Boskabady MH. Animal model of asthma, various methods and measured parameters: a methodological review. Iran J Allergy Asthma Immunol 2016;15:445-65.

26. Rosenberg HF, Druey KM. Modeling asthma: pitfalls, promises, and the road ahead. J Leukoc Biol 2018;104:41-8.

27. Shin YS, Takeda K, Gelfand EW. Understanding asthma using animal models. Allergy Asthma Immunol Res 2009;1:10-8.

28. Przybyszowski M, Paciorek K, Zastrzezynska W, et al. Influence of omalizumab therapy on airway remodeling assessed with high-resolution computed tomography (HRCT) in severe allergic asthma patients. Adv Respir Med 2018. [Epub ahead of print].

29. Kotaru C, Schoonover KJ, Trudeau JB, et al. Regional fibroblast heterogeneity in the lung: implications for remodeling. Am J Respir Crit Care Med 2006;173:1208-15.

30. Witt CA, Sheshadri A, Carlstrom L, et al. Longitudinal changes in airway remodeling and air trapping in severe asthma. Acad Radiol 2014;21:986-93.

31. Durrani SR, Viswanathan RK, Busse WW. What effect does asthma treatment have on airway remodeling? Current perspectives. J Allergy Clin Immunol 2011;128:439-48; quiz 449-50.

32. Yadav SK, Shah SD, Penn RB. Give me a fork: can autophagy research solve the riddle of airway remodeling in asthma? Am J Respir Cell Mol Biol 2019;60:494-6.

33. Vieira Braga FA, Kar G, Berg M, et al. A cellular census of human lungs identifies novel cell states in health and in asthma. Nat Med 2019;25:1153-63.

34. Wu J, Dong F, Wang RA, et al. Central role of cellular senescence in TSLP-induced airway remodeling in asthma. PLoS One 2013;8:e77795.

35. McAlinden KD, Deshpande DA, Ghavami S, et al. Autophagy activation in asthma airways remodeling. Am J Respir Cell Mol Biol 2019;60:541-53.

36. Broide DH. Immunologic and inflammatory mechanisms that drive asthma progression to remodeling. J Allergy Clin Immunol 2008;121:560-70; quiz 571-2.

37. Hur GY, Broide DH. Genes and pathways regulating decline in lung function and airway remodeling in asthma. Allergy Asthma Immunol Res 2019;11:604-21.

38. Gomez JL, Crisafi GM, Holm CT, et al. Genetic variation in chitinase 3-like 1 (CHI3L1) contributes to asthma severity and airway expression of YKL-40. J Allergy Clin Immunol 2015;136:51-8.e10.

39. Ierodiakonou D, Portelli MA, Postma DS, et al. Urokinase plasminogen activator receptor polymorphisms and airway remodelling in asthma. Eur Respir J 2016;47:1568-71.

40. Nakamura Y, Suzuki R, Mizuno T, et al. Therapeutic implication of genetic variants of IL13 and STAT4 in airway remodelling with bronchial asthma. Clin Exp Allergy 2016;46:1152-61.

41. Fuso L, Macis G, Condoluci C, et al. Impulse oscillometry and nitrogen washout test in the assessment of small airway 
dysfunction in asthma: Correlation with quantitative computed tomography. J Asthma 2019;56:323-31.

42. Mostaço-Guidolin LB, Osei ET, Ullah J, et al. Defective fibrillar collagen organization by fibroblasts contributes to airway remodeling in asthma. Am J Respir Crit Care Med 2019;200:431-43.

43. Zhang X, Xia T, Lai Z, et al. Uncontrolled asthma phenotypes defined from parameters using quantitative CT analysis. Eur Radiol 2019;29:2848-58.

44. Brigham EP, West NE. Diagnosis of asthma: diagnostic testing. Int Forum Allergy Rhinol 2015;5 Suppl 1:S27-30.

45. de Blic J, Tillie-Leblond I, Emond S, et al. Highresolution computed tomography scan and airway remodeling in children with severe asthma. J Allergy Clin Immunol 2005;116:750-4.

46. Nishimoto K, Karayama M, Inui N, et al. Relationship between fraction of exhaled nitric oxide and airway morphology assessed by three-dimensional CT analysis in asthma. Sci Rep 2017;7:10187.

47. Jiang D, Wang Z, Yu N, et al. Airway remodeling in asthma: evaluation in 5 consecutive bronchial generations by using high-resolution computed tomography. Respir Care 2018;63:1399-406.

48. Jiang D, Wang Z, Shen C, et al. Small airway dysfunction may be an indicator of early asthma: findings from high-resolution CT. Ann Allergy Asthma Immunol 2019; 122:498-501.

49. Shim SS, Schiebler ML, Evans MD, et al. Lumen area change (Delta Lumen) between inspiratory and expiratory multidetector computed tomography as a measure of severe outcomes in asthmatic patients. J Allergy Clin Immunol 2018;142:1773-80.e9.

50. Adams DC, Hariri LP, Miller AJ, et al. Birefringence microscopy platform for assessing airway smooth muscle structure and function in vivo. Sci Transl Med 2016;8:359ra131.

51. Bahmer T, Sand JMB, Weckmann M. Lost in transition: biomarkers of remodeling in patients with asthma. Curr Opin Pulm Med 2020;26:40-6.

52. Borrebaeck CA. Precision diagnostics: moving towards protein biomarker signatures of clinical utility in cancer. Nat Rev Cancer 2017;17:199-204.

53. Vijverberg SJ, Hilvering B, Raaijmakers JA, et al. Clinical utility of asthma biomarkers: from bench to bedside. Biologics 2013;7:199-210.

54. Stokes JR, Casale TB. Characterization of asthma endotypes: implications for therapy. Ann Allergy Asthma Immunol 2016;117:121-5.
55. Narendra D, Blixt J, Hanania NA. Immunological biomarkers in severe asthma. Semin Immunol 2019;46:101332.

56. Wadsworth S, Sin D, Dorscheid D. Clinical update on the use of biomarkers of airway inflammation in the management of asthma. J Asthma Allergy 2011;4:77-86.

57. Ayars AG, Altman LC, Potter-Perigo S, et al. Sputum hyaluronan and versican in severe eosinophilic asthma. Int Arch Allergy Immunol 2013;161:65-73.

58. Bulut I, Ozseker ZF, Coskun A, et al. Pregnancy-associated plasma protein-A (PAPP-A) levels in patients with severe allergic asthma are reduced by omalizumab. J Asthma 2018;55:1116-21.

59. Kanemitsu Y, Matsumoto H, Izuhara K, et al. Increased periostin associates with greater airflow limitation in patients receiving inhaled corticosteroids. J Allergy Clin Immunol 2013;132:305-12.e3.

60. Rasmussen F, Taylor DR, Flannery EM, et al. Risk factors for airway remodeling in asthma manifested by a low postbronchodilator FEV1/vital capacity ratio: a longitudinal population study from childhood to adulthood. Am J Respir Crit Care Med 2002;165:1480-8.

61. Gupta S, Hartley R, Khan UT, et al. Quantitative computed tomography-derived clusters: redefining airway remodeling in asthmatic patients. J Allergy Clin Immunol 2014;133:729-38.e18.

62. Hoshino M, Takahashi M, Takai Y, et al. Inhaled corticosteroids decrease subepithelial collagen deposition by modulation of the balance between matrix metalloproteinase-9 and tissue inhibitor of metalloproteinase-1 expression in asthma. J Allergy Clin Immunol 1999;104:356-63.

63. Chung FT, Huang HY, Lo CY, et al. Increased ratio of matrix metalloproteinase-9 (MMP-9)/tissue inhibitor metalloproteinase-1 from alveolar macrophages in chronic asthma with a fast decline in FEV1 at 5-year follow-up. J Clin Med 2019;8:1451.

64. Aubier M, Thabut G, Hamidi F, et al. Airway smooth muscle enlargement is associated with protease-activated receptor 2/ligand overexpression in patients with difficultto-control severe asthma. J Allergy Clin Immunol 2016;138:729-39.e11.

65. Hirota N, Martin JG. Mechanisms of airway remodeling. Chest 2013;144:1026-32.

66. de Boer RA, Voors AA, Muntendam P, et al. Galectin-3: a novel mediator of heart failure development and progression. Eur J Heart Fail 2009;11:811-7.

67. Meijers WC, van der Velde AR, Pascual-Figal DA, et 
al. Galectin-3 and post-myocardial infarction cardiac remodeling. Eur J Pharmacol 2015;763:115-21.

68. Ge XN, Bahaie NS, Kang BN, et al. Allergen-induced airway remodeling is impaired in galectin-3-deficient mice. J Immunol 2010;185:1205-14.

69. Mauri P, Riccio AM, Rossi R, et al. Proteomics of bronchial biopsies: galectin-3 as a predictive biomarker of airway remodelling modulation in omalizumab-treated severe asthma patients. Immunol Lett 2014;162:2-10.

70. Riccio AM, Mauri P, De Ferrari L, et al. Galectin-3: an early predictive biomarker of modulation of airway remodeling in patients with severe asthma treated with omalizumab for 36 months. Clin Transl Allergy 2017;7:6.

71. Hara A, Niwa M, Noguchi K, et al. Galectin-3 as a nextgeneration biomarker for detecting early stage of various diseases. Biomolecules 2020;10:389.

72. Tong X, Wang D, Liu S, et al. The YKL-40 protein is a potential biomarker for COPD: a meta-analysis and systematic review. Int J Chron Obstruct Pulmon Dis 2018;13:409-18.

73. Lee CG, Da Silva CA, Dela Cruz CS, et al. Role of chitin and chitinase/chitinase-like proteins in inflammation, tissue remodeling, and injury. Annu Rev Physiol 2011;73:479-501.

74. Bara I, Ozier A, Girodet PO, et al. Role of YKL-40 in bronchial smooth muscle remodeling in asthma. Am J Respir Crit Care Med 2012;185:715-22.

75. Konradsen JR, James A, Nordlund B, et al. The chitinaselike protein YKL-40: a possible biomarker of inflammation and airway remodeling in severe pediatric asthma. J Allergy Clin Immunol 2013;132:328-35.e5.

76. Chupp GL, Lee CG, Jarjour N, et al. A chitinase-like protein in the lung and circulation of patients with severe asthma. N Engl J Med 2007;357:2016-27.

77. Furuhashi K, Suda T, Nakamura Y, et al. Increased expression of YKL-40, a chitinase-like protein, in serum and lung of patients with idiopathic pulmonary fibrosis. Respir Med 2010;104:1204-10.

78. Brandi ML, Minisola S. Calcidiol [25(OH)D3]: from diagnostic marker to therapeutical agent. Curr Med Res Opin 2013;29:1565-72.

79. Jiao J, Castro M. Vitamin D and asthma: current perspectives. Curr Opin Allergy Clin Immunol 2015;15:375-82.

80. Damera G, Fogle HW, Lim P, et al. Vitamin D inhibits growth of human airway smooth muscle cells through growth factor-induced phosphorylation of retinoblastoma protein and checkpoint kinase 1. Br J Pharmacol
2009;158:1429-41.

81. Gupta A, Sjoukes A, Richards D, et al. Relationship between serum vitamin $\mathrm{D}$, disease severity, and airway remodeling in children with asthma. Am J Respir Crit Care Med 2011;184:1342-9.

82. Das G, Taylor PN, Javaid H, et al. Seasonal variation of vitamin $\mathrm{D}$ and serum thyrotropin levels and its relationship in a euthyroid Caucasian population. Endocr Pract 2018;24:53-9.

83. Kim SM, Choi HJ, Lee JP, et al. Prevalence of vitamin $\mathrm{D}$ deficiency and effects of supplementation with cholecalciferol in patients with chronic kidney disease. J Ren Nutr 2014;24:20-5.

84. Niimi A, Matsumoto H, Amitani R, et al. Effect of shortterm treatment with inhaled corticosteroid on airway wall thickening in asthma. Am J Med 2004;116:725-31.

85. Boulet LP, Turcotte H, Laviolette M, et al. Airway hyperresponsiveness, inflammation, and subepithelial collagen deposition in recently diagnosed versus longstanding mild asthma. Influence of inhaled corticosteroids. Am J Respir Crit Care Med 2000;162:1308-13.

86. Chakir J, Shannon J, Molet S, et al. Airway remodelingassociated mediators in moderate to severe asthma: effect of steroids on TGF-beta, IL-11, IL-17, and type I and type III collagen expression. J Allergy Clin Immunol 2003;111:1293-8.

87. Royce SG, Li X, Tortorella S, et al. Mechanistic insights into the contribution of epithelial damage to airway remodeling. Novel therapeutic targets for asthma. Am J Respir Cell Mol Biol 2014;50:180-92.

88. Fang L, Wang X, Sun Q, et al. IgE downregulates PTEN through microRNA-21-5p and stimulates airway smooth muscle cell remodeling. Int J Mol Sci 2019;20:875.

89. Roth M, Zhao F, Zhong J, et al. Serum IgE induced airway smooth muscle cell remodeling is independent of allergens and is prevented by omalizumab. PLoS One 2015;10:e0136549.

90. Hoshino M, Ohtawa J. Effects of adding omalizumab, an anti-immunoglobulin $\mathrm{E}$ antibody, on airway wall thickening in asthma. Respiration 2012;83:520-8.

91. Brightling CE, Chanez P, Leigh R, et al. Efficacy and safety of tralokinumab in patients with severe uncontrolled asthma: a randomised, double-blind, placebo-controlled, phase 2b trial. Lancet Respir Med 2015;3:692-701.

92. Hanania NA, Noonan M, Corren J, et al. Lebrikizumab in moderate-to-severe asthma: pooled data from two randomised placebo-controlled studies. Thorax 2015;70:748-56. 
93. Saglani S, Lui S, Ullmann N, et al. IL-33 promotes airway remodeling in pediatric patients with severe steroidresistant asthma. J Allergy Clin Immunol 2013;132:67685.e13.

94. Castro M, Rubin AS, Laviolette M, et al. Effectiveness and safety of bronchial thermoplasty in the treatment of severe asthma: a multicenter, randomized, double-blind, sham-controlled clinical trial. Am J Respir Crit Care Med 2010;181:116-24.

95. Thomson NC. Recent developments in bronchial thermoplasty for severe asthma. J Asthma Allergy 2019;12:375-87.

96. Pretolani M, Bergqvist A, Thabut G, et al. Effectiveness of bronchial thermoplasty in patients with severe refractory asthma: clinical and histopathologic correlations. J Allergy Clin Immunol 2017;139:1176-85.

97. Chernyavsky IL, Russell RJ, Saunders RM, et al. In vitro, in silico and in vivo study challenges the impact of bronchial thermoplasty on acute airway smooth muscle mass loss. Eur Respir J 2018;51:1701680.

98. Matsuse H, Yamagishi T, Kodaka N, et al. Tiotropium bromide as add-on therapy to inhaled corticosteroids for treating asthma. Expert Opin Pharmacother 2015;16:1403-9.

99. Bullone M, Vargas A, Elce Y, et al. Fluticasone/salmeterol reduces remodelling and neutrophilic inflammation in severe equine asthma. Sci Rep 2017;7:8843.

100. Girodet PO, Dournes G, Thumerel M, et al. Calcium channel blocker reduces airway remodeling in severe asthma. A proof-of-concept study. Am J Respir Crit Care Med 2015;191:876-83.

101. Trian T, Benard G, Begueret H, et al. Bronchial smooth muscle remodeling involves calcium-dependent enhanced mitochondrial biogenesis in asthma. J Exp Med 2007;204:3173-81.

102. White MV. The role of histamine in allergic diseases. J Allergy Clin Immunol 1990;86:599-605.

103. Wilson AM. The role of antihistamines in asthma

Cite this article as: Zhang J, Dong L. Status and prospects: personalized treatment and biomarker for airway remodeling in asthma. J Thorac Dis 2020;12(10):6090-6101. doi: 10.21037/ jtd-20-1024 management. Treat Respir Med 2006;5:149-58.

104. Kunzmann S, Schmidt-Weber C, Zingg JM, et al. Connective tissue growth factor expression is regulated by histamine in lung fibroblasts: potential role of histamine in airway remodeling. J Allergy Clin Immunol 2007;119:1398-407.

105. Simons FE. Advances in H1-antihistamines. N Engl J Med 2004;351:2203-17.

106. Paggiaro P, Bacci E. Montelukast in asthma: a review of its efficacy and place in therapy. Ther Adv Chronic Dis 2011;2:47-58.

107.Hung CH. Role of leukotriene receptor antagonists in asthma. Pediatr Neonatol 2012;53:219-20.

108. Kelly MM, Chakir J, Vethanayagam D, et al. Montelukast treatment attenuates the increase in myofibroblasts following low-dose allergen challenge. Chest 2006;130:741-53.

109. Gonem S, Berair R, Singapuri A, et al. Fevipiprant, a prostaglandin D2 receptor 2 antagonist, in patients with persistent eosinophilic asthma: a single-centre, randomised, double-blind, parallel-group, placebocontrolled trial. Lancet Respir Med 2016;4:699-707.

110.Saunders R, Kaul H, Berair R, et al. DP2 antagonism reduces airway smooth muscle mass in asthma by decreasing eosinophilia and myofibroblast recruitment. Sci Transl Med 2019;11:eaao6451.

111. Canonica GW, Ferrando M, Baiardini I, et al. Asthma: personalized and precision medicine. Curr Opin Allergy Clin Immunol 2018;18:51-8.

112. Siddiqui S, Shikotra A, Richardson M, et al. Airway pathological heterogeneity in asthma: Visualization of disease microclusters using topological data analysis. J Allergy Clin Immunol 2018;142:1457-68.

113. Brasier AR, Zhou J. Validation of the epigenetic reader bromodomain-containing protein 4 (BRD4) as a therapeutic target for treatment of airway remodeling. Drug Discov Today 2020;25:126-32. 